\title{
Nonstructural protein 11 (nsp11) of porcine reproductive and respiratory syndrome virus (PRRSV) promotes PRRSV infection in MARC-145 cells
}

Xibao Shi ${ }^{1,2^{* \dagger}}$, Xiaozhuan Zhang ${ }^{1,3+}$, Yongzhe Chang ${ }^{2}$, Bo Jiang ${ }^{5}$, Ruiguang Deng ${ }^{2}$, Aiping Wang ${ }^{4}$ and Gaiping Zhang ${ }^{2,3^{*}}$

\begin{abstract}
Background: Porcine reproductive and respiratory syndrome virus (PRRSV) induces one of most important devastating disease of swine worldwide, and the current methods poorly control it. Previous studies have indicated that the nonstructural protein 11 (nsp11) of PRRSV may be an important protein for the immune escape of PRRSV.

Results: Here, we firstly explored the effect of over-expression of nsp11 on PRRSV infection and found that over-expression of nsp11 enhanced the PRRSV titers while the small interfering RNA (siRNAs) specifically targeting nsp11 could reduce the PRRSV titers in MARC-145 cells.
\end{abstract}

Conclusion: In conclusion, PRRSV nsp11 promotes PRRSV infection in MARC-145 cells and siRNAs targeting nsp11 may be a potential therapeutic strategy to control PRRSV in future.

Keywords: PRRSV, Small interfering RNA, nsp11

\section{Background}

PRRSV, a positive sense and single-stranded RNA virus, is a member of family Arteriviridae [1]. Since it was emerged in the United States in 1987 and in Europe in 1990, PRRSV has rapidly spread in the swine producing regions and became one of the most important devastating diseases of swine worldwide. It can cause severe reproductive failure in sows and respiratory distress in young growing pigs [2]. Infection with PRRSV also made pigs easy to secondary infection by other pathogens [3]. Up to date, since there is no efficient method or drugs against PRRSV, it is very important and urgent to develop the effective therapeutic strategies to control PRRS.

\footnotetext{
* Correspondence: shixibao@aliyun.com; zhanggaiping2003@163.com ${ }^{\dagger}$ Equal contributors

${ }^{1}$ College of Life Sciences, Henan Normal University, Xinxiang 453007, China

${ }^{2}$ Key Laboratory of Animal Immunology of the Ministry of Agriculture, Henan Provincial Key Laboratory of Animal Immunology, Henan Academy of

Agricultural Sciences, Zhengzhou, Henan 450002, China

Full list of author information is available at the end of the article
}

The PRRSV genome has nine open reading frames (ORFs) composed of ORF1a, ORF1b, ORF2a, ORF2b, and ORF3-7. ORF1a and ORF1b could produce 16 nonstructural proteins (nsp1 $\alpha$, nsp1 $\beta$, nsp2 etc.) [4-7]. Previous studies have shown that the nsp11 of equine arteritis virus(EAV), which is another member of family Arteriviridae, may play a key role in viral RNA synthesis and additional functions in the viral life cycle [8]. Other and our previous work also demonstrated that PRRSV nsp11 inhibited the host innate immune responses such as the transcription of type I interferon [7], the RNAi innate immune response [9] and the NLR family pyrin domain-containing 3 (NLRP3)-mediated production of IL-1 $[10]$, which indicated that PRRSV nsp11 may play an important role in PRRSV infection. So the purpose of present study is to explore the effect of over-expression of nsp11 on PRRSV infection and whether the siRNAs targeting the PRRSV nsp11 could influence PRRSV infection. 


\section{Methods}

\section{Cell and virus}

MARC-145 cells, derived from a monkey fibroblast cell line MA-104 [11], and 293T cells were grown in Dulbecco's Modified Eagle medium (Gibco) plus 10 \% heatinactivated fetal bovine serum (Hyclone). PRRSV strain BJ-4 was a kind gift from Prof. Hanchun Yang (China Agricultural University).

\section{Plasmids}

The pcDNA3.1-GFP-nsp11 plasmid was constructed by sub-cloning from the plasmid pcDNA3.1-FLAG-nsp11 [7] to pcDNA3.1-GFP [12] using the restriction endonuclease Hind III and EcoRI. The plasmids pcDNA3.1-FLAG, pcDNA3.1-FLAG-nsp11 and pcDNA3.1-FLAG-nsp11 H129A have been described in our previous work [7].

\section{Transfection of plasmids and viral infection}

All newly-prepared plasmids were confirmed correctly by DNA sequencing. Transient transfection was carried out by using Lipofectamine 2000 (Invitrogen). Cells cultured in 24-well plates were transfected with the indicated expression plasmid or control vector (800 ng/well) in triplicate. And $6 \mathrm{~h}$ (h) later, the cells were infected with PRRSV at a multiplicity of infection (MOI) of 0.1 , and then the cells were lysed by freezing and thawing three times after $24 \mathrm{~h}$ infection. The supernatants were collected by centrifugation, and the viral titers were detected by $50 \%$ tissue culture infected dose (TCID50) assay using the method of Reed-Muench in the MARC-145 cells.

\section{Western blots}

The $293 \mathrm{~T}$ cells were cultured in 24-well plates and transfected with pcDNA3.1-GFP-nsp11 or pcDNA3.1GFP and nsp11 siRNA (100 nM) or control siRNA $(100 \mathrm{nM})$ in triplicate, and $36 \mathrm{~h}$ later, the cells were lysed with lysing buffer ( $1 \%$ Nonidet P-40, $0.1 \%$ sodium deoxycholate, $0.1 \%$ SDS, 50 mM Tris- $\mathrm{HCl}$ $(\mathrm{pH}$ 7.4), $150 \mathrm{mM} \mathrm{NaCl}, 2 \mathrm{mM}$ EDTA, $2 \mathrm{mM}$ Na3VO4, $2 \mathrm{mM} \mathrm{NaF}$ and a protease inhibitor cocktail). The detailed procedure for immunoblots has been described in our previous work [13]. Briefly, the proteins were separated by $10 \%$ SDS-PAGE and transferred to polyvinylidene difluoride (PVDF) membranes (Millipore Company, Boston, Massachusetts, USA), and then the PVDF membranes were incubated with anti-GFP (Clontech) or anti-actin (Cell Signaling Technology) antibodies. Subsequently the membranes were incubated with appropriate secondary antibodies and were tested by an ECL detection system (Cell Signaling Technology, Boston, USA).

\section{Transfection of siRNA and viral infection}

Cells cultured in 24-well plates were transfected with the indicated siRNA in triplicate $(100 \mathrm{nM} /$ well) (chemical synthesis by Bioneer) (Table. 1). And $6 \mathrm{~h}$ later, the cells were infected with PRRSV strain BJ-4 at an MOI of 0.1 , and $24 \mathrm{~h}$ later, the cells were lysed by freezing and thawing three times. The supernatants were collected by centrifugation, and the viral titers were detected by TCID50 using the method of Reed-Muench in the MARC-145 cells.

\section{Real time (RT)-PCR}

MARC-145 cells cultured in 24-well plates were transfected in triplicate with nsp11-siRNAs/control siRNA and plasmid pcDNA3.1-GFP-nsp11 (800 ng/well) or pcDNA 3.1-GFP (800 ng/well). And $24 \mathrm{~h}$ later, the cells were infected with PRRSV at an MOI of 0.1, and $48 \mathrm{~h}$ later, the cellular RNA was extracted with TRIzol (Invitrogen). M-MLV reverse transcriptase was used for the PrimeScript $^{\mathrm{Tm}}$ RT Reagent Kit with gDNA Eraser (Takara, Dalian, China). Quantitative real-time RT-PCR (qRT-PCR) was performed using SYBR $^{\circledast}$ Premix Ex $\mathrm{Taq}^{\mathrm{Tm}}$ (Takara, Dalian, China) and was tested by the 7500 First real-time PCR system (Applied Biosystems, Foster City, CA, USA). Glyceraldehyde-3-phosphate dehydrogenase (GAPDH) was used as an internal control. The $2^{-\Delta \Delta \mathrm{Ct}}$-method was used to analyze the relative amount of target gene expression.

Table 1 Synthesized siRNAs sequences targeting the regions of PRRSV nsp11 and the primers of ORF-7 for RT-PCR

\begin{tabular}{|c|c|c|c|}
\hline Gene of target & Name of siRNA & Location(bp) & Sequence $\left(5^{\prime}-3^{\prime}\right)$ \\
\hline nsp 11 & nsp11 siRNA 1 & $244-262$ & CGTGTCATACTATCTCACA \\
\hline nsp 11 & nsp11 siRNA 2 & $526-544$ & CACACTGACAGATGTGTAC \\
\hline nsp 11 & nsp11 siRNA 3 & $409-427$ & CACTACCGTTGGAGGATGT \\
\hline Negative control & Control siRNA & & CCTACGCCACCAATTTCGT \\
\hline ORF-7 For & & & AAACCAGTCCAGAGGCAAGG \\
\hline ORF-7 Rev & & & GCAAACTAAACTCCACAGTGTAA \\
\hline GAPDH For & & & TGACAACAGCCTCAAGATCG \\
\hline GAPDH Rev & & & GTCTTCTGGGTGGCAGTGAT \\
\hline
\end{tabular}




\section{Viral titers}

MARC-145 cells transfected with the nsp11 siRNA (100 $\mathrm{nM})$ or control siRNA (100 $\mathrm{nM})$ and the plasmid pcDNA3.1-GFP-nsp11 (800 ng/well) or pcDNA3.1-GFP (800 ng/well), and $24 \mathrm{~h}$ later, the cells were infected by PRRSV at an MOI of 0.1, the cells were frozen and thawed in three cycles and collected after $48 \mathrm{~h}$ infection. Then the viral titers were determined by TCID50 assay using the method of Reed-Muench in the cells of MARC-145.

\section{Statistical analysis}

Statistical analyses were performed by Student's t-test, and the differences were considered as statistical significance when $P<0.05$.

\section{Results}

Over-expression of nsp11 enhanced PRRSV titers in MARC-145 cells

Firstly, we successfully constructed the expression plasmid of pcDNA 3.1-GFP-nsp11 (GFP-nsp11) and the western blot in Fig. 1a confirmed the successful expression of GFP-nsp11 (Fig. 1). Secondly, the MARC-145 cells were cultured in 24-well plates overnight, and then the cells were transfected with the expression plasmid pcDNA3.1-GFP-nsp11 or the control plasmid pcDNA3.1-GFP. The results in Fig. 1b showed that the PRRSV titers from the MARC-145 cells transfected with pcDNA3.1-GFP-nsp11 were about one point six times to that from MARC-145 cells transfected with control plasmid, while the RNA levels of PRRSV from the MARC-145 cells transfected with pcDNA3.1-GFP-nsp11 were three times to that from MARC-145 cells transfected with control plasmid (Fig. 1c).

\section{siRNAs targeting nsp11 reduced PRRSV titers in MARC-145 cells}

Now that over-expression of nsp11 could enhance PRRSV titers, it was reasonable to design siRNAs targeting nsp11 to investigate whether the siRNAs could reduce PRRSV titers. The sequences of the siRNA special for nsp11 and the control siRNA were listed in Table 1. 293T cells (Fig. 2a) or MARC-145 cells (Fig. 2b) grown in 24-well plates were co-transfected with the nsp11 siRNA (100 nM/well) or control siRNA and the plasmid GFP-nsp11 (800 ng/well).

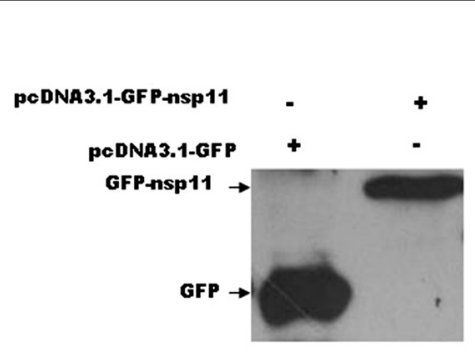

A

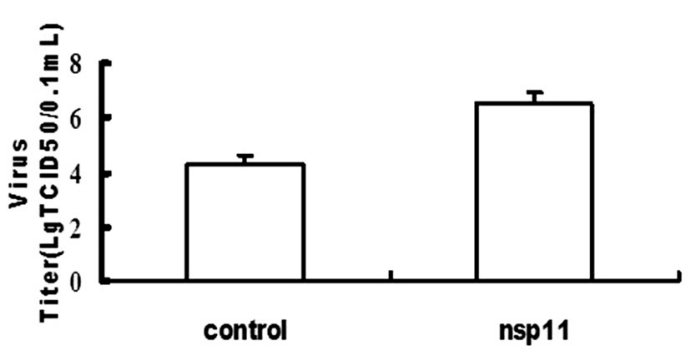

B

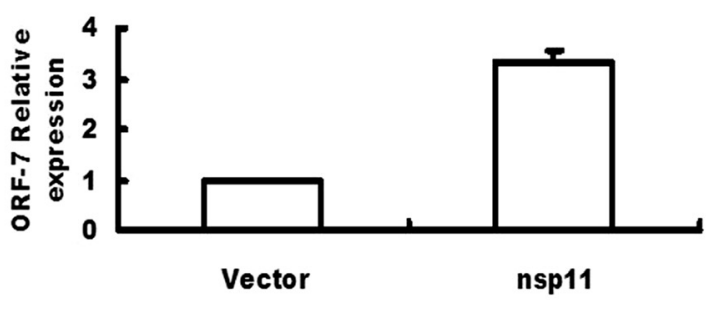

C

Fig. 1 Over-expression of nsp11enhenced the titers of PRRSV. a 293T cells were transfected with pcDNA3.1-GFP (GFP) or pcDNA3.1-GFP-nsp11 (GFP-nsp1 1), and $48 \mathrm{~h}$ later, the cells were analyzed by western blots. b MARC-145 cells cultured in 24-well plate were transfected with pcDNA3.1-GFPnsp1 1 (nsp11)(800 ng/well) or pcDNA 3.1-GFP (Con). And $6 \mathrm{~h}$ later, the cells were infected with PRRSV at an MOI of 0.1 or mock infected, and $24 \mathrm{~h}$ later, the cells were lysed by freezing and thawing three times in three cycles, then the viral titers were measured by TCID50. c MARC-145 cells cultured in 24-well plate were transfected with pcDNA 3.1-GFP-nsp11 (nsp11) (800 ng/well) or pcDNA3.1-GFP (Con). And $6 \mathrm{~h}$ later, the cells were infected with PRRSV at an MOI of 0.1 or mock infected, and $24 \mathrm{~h}$ later, the cells were collected and the viral RNA levels were measured by RT-PCR. Data represented means of three replicates, and experiments were repeated three times. Error bars represented the standard deviations. *: $P<0.05$ compared with the results in control. MOl: multiplicity of infection 


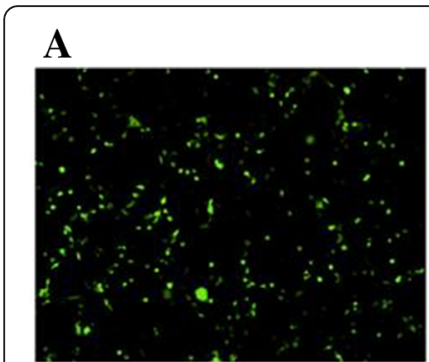

peDNA3.1-GFP+control siRNA

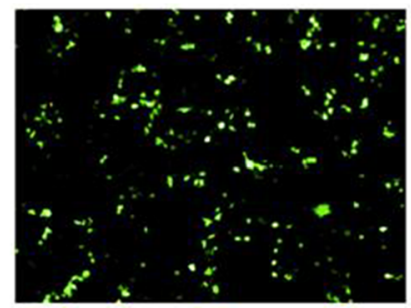

peDNA3.1-nsp11-GFP+control siRNA

B

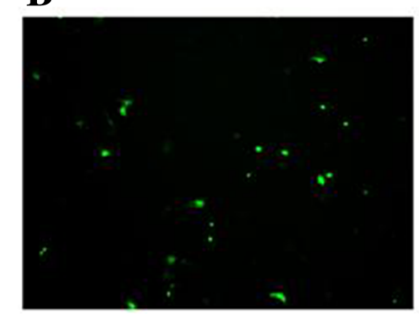

peDNA3.1-GFP+control siRNA

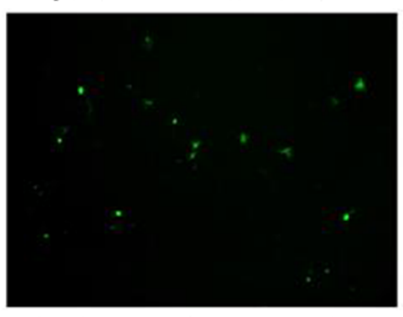

pcDNA3.1-nspll-GFP+conrrol siRNA

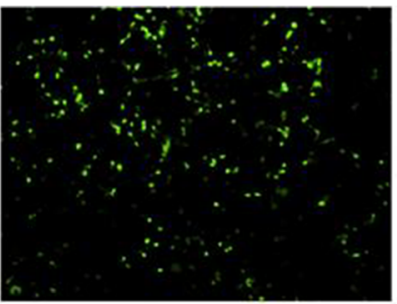

peDNA3.1-GFP+siRNA1

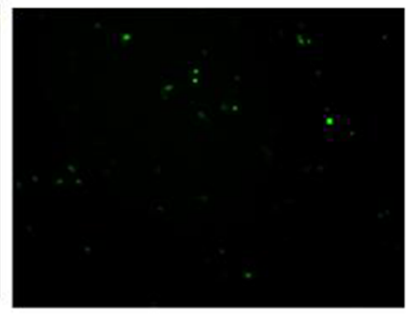

pcDNA3.1-nsp11-GFP+siRNA1

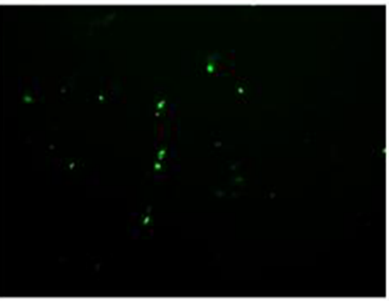

peDNA3.1-GFP+siRNA1

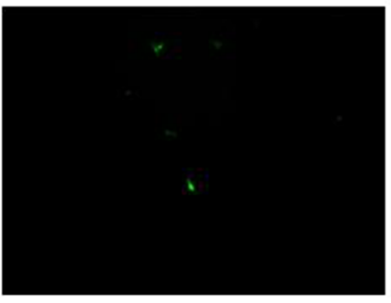

peDNA3.1-nspll-GFP+siRNA 1

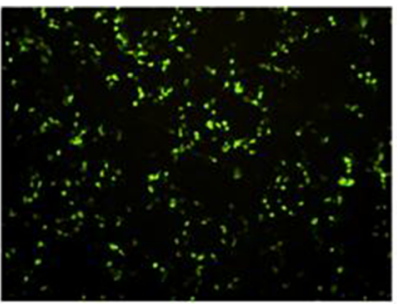

pCDNA3.1-GFP+siRNA2

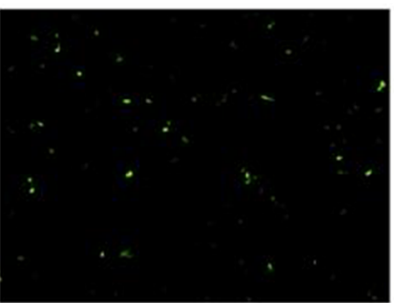

pcDNA3.1-nsp11-GFP+siRNA2

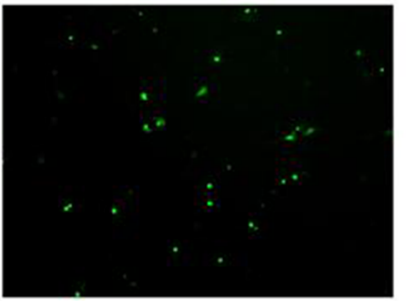

pcDNA3.1-GFP+siRNA2

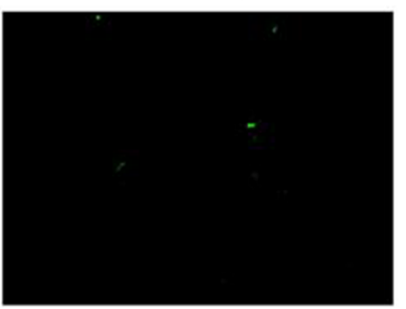

peDNA3.1-nspll-GFP+siRNA 2

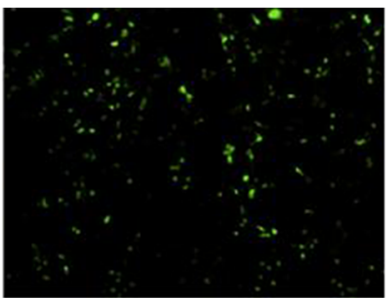

pCDNA3.1-GFP+siRNA3

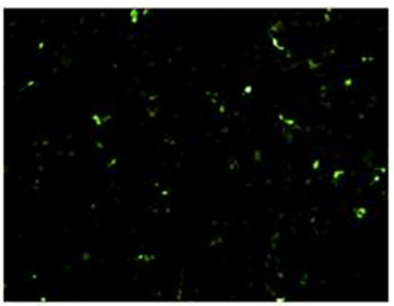

pcDNA3.1-nsp11-GFP+siRNA3

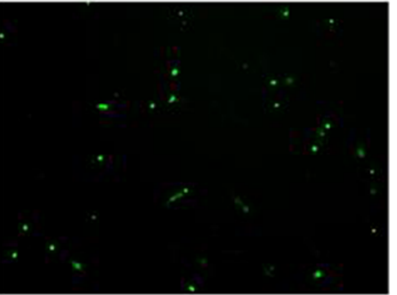

peDNA3.1-GFP+siRNA3

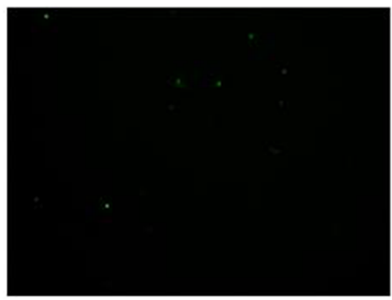

peDNA3.1-nsp11-GFP+siRNA 3

Fig. 2 siRNAs targeting nsp11 could inhibit the expression of GFP- nsp11 and didn't influence on the expression of GFP in 293T cells or in MARC-145 cells. 293T cells (a) or MARC-145 cells (b) cultured in 24-well plates were co-transfected with pcDNA 3.1-GFP-nsp11(800 ng/well) or pcDNA3.1-GFP (800 ng/well) and nsp1 1 siRNA 1 (100 nM), nsp11 siRNA 2 (100 nM), nsp11 siRNA 3(100 nM) or control siRNA (100 nM). And 24 h later, the cells were analyzed by fluorescence microscopy (50X). Data represented means of three replicates, and experiments were repeated three times

And $24 \mathrm{~h}$ later, the cells in five random fields were analyzed by fluorescence microscopy $(50 \times)$ and only one of them was shown in Fig. 2. The results in Fig. 2 showed that all of the three siRNAs targeting nsp11 could inhibit the expression of GFP-nsp11 and didn't influence on the expression of GFP.

Finally, we selected two siRNAs (siRNA1 and siRNA2) targeting nsp11 to determine whether siRNAs targeting nsp11 could reduce PRRSV titers in MARC-145 cell. The western blots results in Fig. 3a confirmed that siRNA1 and siRNA2 could efficiently reduce the nsp11 expression in 293T cells (Fig. 3a). The results in Fig. $3 \mathrm{~b}$ and $\mathrm{c}$ showed that siRNAs targeting nucleic acid sequence of nsp11 could significantly reduce viral titers of PRRSV (Fig. 3b) and reduce RNA levels of PRRSV (Fig. 3c).

The endoribonuclease activity of nsp11 was important for nsp11 to enhance the PRRSV titers

Our previous work has shown that the endoribonuclease activity of nsp11 was important for nsp11 to inhibit the transcription of IFN- $\beta$ [7] and the secretion of IL-1 $\beta$ 
A

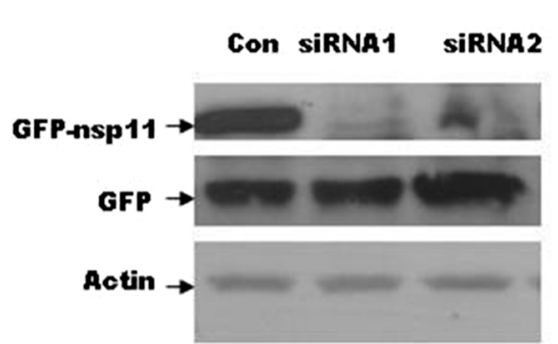

B

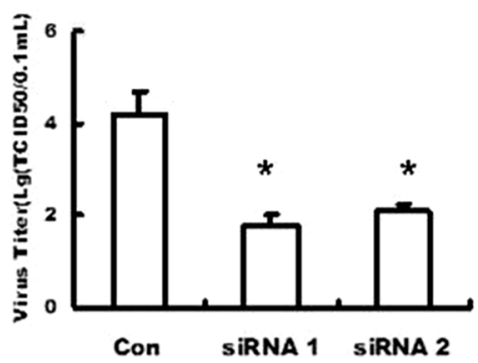

C

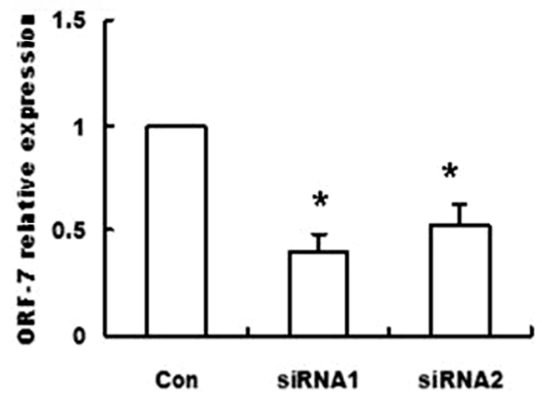

Fig. 3 siRNAs targeting nsp11 could efficiently reduce the titers of PRRSV. a 293T cells grown in 24-well plates were transfected with pcDNA3.1GFP-nsp11(800 ng/well) or pcDNA3.1-GFP (800 ng/well) and nsp11 siRNA 1 (100 nM), nsp11 siRNA 2 (100 nM) or control siRNA (100 nM), then $36 \mathrm{~h}$ later, the cells for collected for the western blot. b MARC-145 cells cultured in 24-well plates were transfected with nsp11 siRNA 1 (100 nM), nsp11 siRNA 2 (100 nM) or control siRNA (100 nM). And 6 h later, the cells were infected with PRRSV at an MOI of 0.1 or mock infected, and $24 \mathrm{~h}$ later, the cells were lysed by freezing and thawing three times, then the viral titers were measured by TCID50. c MARC-145 cells grown in 24-well plates were transfected with nsp11 siRNA 1 (100 nM), nsp11 siRNA 2 (100 nM) or control siRNA (100 nM). And 6 h later, the cells were infected with PRRSV at an $\mathrm{MOI}$ of 0.1 or mock infected, and $24 \mathrm{~h}$ later, the cells were collected and the viral RNA levels were measured by RT-PCR. Data represented means of three replicates, and experiments were repeated three times. Error bars represented the standard deviations. ${ }^{*}$ : $<0.05$ compared with the results in control. MOl, multiplicity of infection

[10]. So next we investigated whether the endoribonuclease activity of nsp11 was important for nsp11 to promote the PRRSV infection. Nedialkova et al. showed that His-129, His-144, and Lys-173 were the catalytic centers, and mutating one of the three amino acids could abolish its enzyme activity. The results in Fig. 4 showed that inactivating the endoribonuclease activity made nsp11 not promote the PRRSV infection.

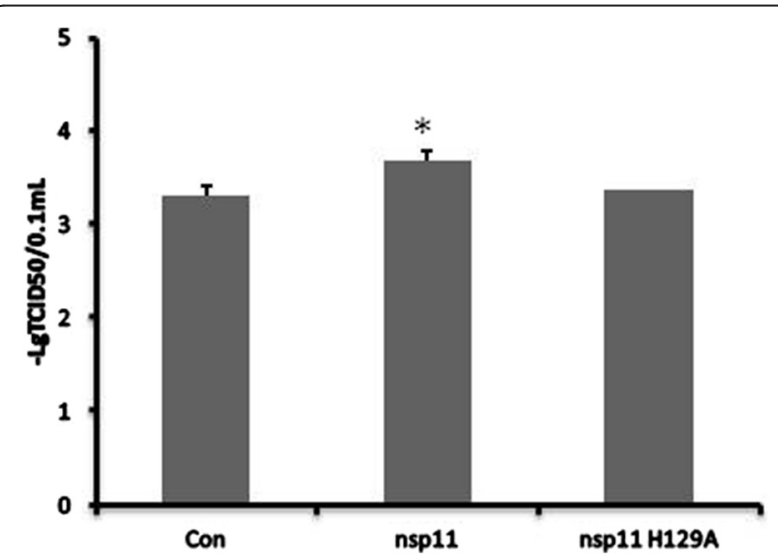

Fig. 4 The endoribonuclease activity of nsp11 was important for nsp11 to enhance the PRRSV titers. MARC-145 cells cultured in 24-well plate were transfected with pcDNA3.1-FLAG-nsp11 (nsp11) (800 ng/well), pcDNA3.1-FLAG-nsp11 H129A (nsp11 H129A) (800 ng/ well) or pcDNA 3.1-FLAG (Con). And $6 \mathrm{~h}$ later, the cells were infected with PRRSV at an $\mathrm{MOI}$ of 0.1 or mock infected, and $24 \mathrm{~h}$ later, the cells were lysed by freezing and thawing three times in three cycles, then the viral titers were measured by TCID50. Data represented means of three replicates, and experiments were repeated three times. Error bars represented the standard deviations. * $P<0.05$ compared with the results in control. $\mathrm{MOl}$, multiplicity of infection

\section{Discussion}

nsp11 was a multi-functional protein. Both our and other previous studies have shown that PRRSV nsp11 is an interferon antagonist [7, 14] and that nsp11 plays an important role in viral RNA synthesis and in the viral life cycle [8]. Our recent work also demonstrated that PRRSV nsp11 inhibited the RNAi innate immune response [9] and NLRP3-mediated production of IL-1 $\beta$ [10]. While our present work showed that over expression of PRRSV nsp11 could enhance the titers of PRRSV (Fig. 1), so our present work gave a directly evidence that nsp11 was an important viral component for upregulating the PRRSV titers.

Identification of and targeting viral important components is useful for developing viral vaccine and controlling the virus. For example, both the nonstructural protein 1 of influenza virus and the nonstructural protein 1 of mouse hepatitis virus (MHV) were important for the viral virulence respectively, and both the modified live-viral vaccines that deletion of nonstructural protein 1 resulted in complete protection against challenge with influenza virus infection and MHV infection respectively [15-17].

RNA interference (RNAi) is an exciting method to silence viral genes. Inhibition of specific genes by siRNA has proven to be a potential therapeutic strategy against viral infection [18], especially for the positive single stranded RNA viruses since their genomes function as both the mRNA and the replication template $[19,20]$. Up to date, RNAi has been used against several viruses such as hepatitis B virus, foot-and-mouth disease virus, dengue virus and so on [20-22]. In this work, we also explore whether the siRNA, which targeted the nucleic acid sequence of nsp11, influenced the titers of PRRSV, 
and the results showed that siRNA targeting nsp11 significantly reduced the titers of PRRSV (Fig. 3). A recent improved live PRRSV vaccine has indicated that the ORF1a and ORF1b were the virulence determinants of PRRSV [23]. In addition, our recent work also show that RNAi innate immune response was an antiviral response to PRRSV and PRRSV inhibited this response by PRRSV nsp1 $\alpha$ and nsp11, which indicated that targeting nsp11 would be useful for RNAi innate immunity against PRRSV [9]. So it is reasonable to propose that our present results gave a new clue for generating the new PRRSV vaccine by targeting PRRSV nsp11.

\section{Conclusion}

In conclusion, our present study has shown that nsp 11 was an important viral component for up-regulating the titers of PRRSV and that siRNAs directly targeting nsp11 could inhibit PRRSV infection, which indicated that PRRSV nsp11 may be an interesting target for controlling PRRSV in future.

\section{Abbreviations}

EAV, equine arteritis virus; GAPDH, Glyceraldehyde-3-phosphate dehydrogenase; GFP-nsp11, pcDNA 3.1-GFP-nsp11; MHV, mouse hepatitis virus; MOI, multiplicity of infection; NLRP3, NLR family pyrin domain-containing 3; nsp11, nonstructural protein 11; ORF, open reading frame; PRRSV, porcine reproductive and respiratory syndrome virus; PVDF, polyvinylidene difluoride; GRT-PCR, quantitative real-time RT-PCR; RNAi, RNA interference; siRNA, small interfering RNA; TCID50, $50 \%$ tissue culture infected dose

\section{Acknowledgements}

We would like to thank Prof. Hanchun Yang for providing PRRSV strain BJ-4.

\section{Funding}

This work was supported by the National Natural Science Foundation of China (grant no. 31302073), the key project of National Natural Science Fund (No.31490600), a grant from the Major State Basic Research Development Program of China (973 Program) (No.2014CB542700), the key scientific research projects of Henan provincial institution of higher education(16A180008), the Doctoral Starting up Foundation of Henan Normal University (5101049170153) and another National Foundation of China (grant no. 31472177), Natural Science Foundation Project of CQ CSTC(cstc2012jjA10108).

\section{Availability of data and materials}

All datasets are available in the main manuscript.

\section{Authors' contributions}

SX, ZX, ZG designed the study, SX, ZX and CY performed the experiments and wrote the paper, and WA, JB and DR performed statistical analysis. All authors read and approved the final manuscript.

\section{Competing interests}

The authors declare that they have no competing interests.

\section{Consent for publication}

Our present work does not contain any individual persons' data, so it is not applicable.

\section{Ethics approval and consent to participate}

Our present work didn't use animals.

\section{Author details}

${ }^{1}$ College of Life Sciences, Henan Normal University, Xinxiang 453007, China.

${ }^{2}$ Key Laboratory of Animal Immunology of the Ministry of Agriculture, Henan
Provincial Key Laboratory of Animal Immunology, Henan Academy of Agricultural Sciences, Zhengzhou, Henan 450002, China. ${ }^{3}$ College of Veterinary Medicine and Animal Science, Henan Agricultural University, Zhengzhou, Henan 450002, China. ${ }^{4}$ Department of Bioengineering, Zhengzhou University, Zhengzhou, Henan 450000, 450002, China. ${ }^{5}$ Office of Science \& Technology, Chongqing Police College, Chongqing 401331, China.

Received: 16 November 2015 Accepted: 1 June 2016

Published online: 06 June 2016

\section{References}

1. Cavanagh D. Nidovirales: a new order comprising Coronaviridae and Arteriviridae. Arch Virol. 1997;142(3):629-33.

2. Zimmerman JJ, Yoon KJ, Wills RW, Swenson SL. General overview of PRRSV: a perspective from the United States. Vet Microbiol. 1997;55(1-4):187-96.

3. Mateu E, Diaz I. The challenge of PRRS immunology. Vet J. 2008;177(3):345-51.

4. den Boon JA, Snijder EJ, Chirnside ED, de Vries AA, Horzinek MC, Spaan WJ. Equine arteritis virus is not a togavirus but belongs to the coronaviruslike superfamily. J Virol. 1991;65(6):2910-20.

5. Chen Z, Lawson S, Sun Z, Zhou X, Guan X, Christopher-Hennings J, Nelson EA, Fang $Y$. Identification of two auto-cleavage products of nonstructural protein 1 (nsp1) in porcine reproductive and respiratory syndrome virus infected cells: nsp1 function as interferon antagonist. Virology. 2010;398(1): 87-97.

6. den Boon JA, Faaberg KS, Meulenberg JJ, Wassenaar AL, Plagemann PG, Gorbalenya AE, Snijder EJ. Processing and evolution of the N-terminal region of the arterivirus replicase ORF1a protein: identification of two papainlike cysteine proteases. J Virol. 1995;69(7):4500-5.

7. Shi X, Wang L, Li X, Zhang G, Guo J, Zhao D, Chai S, Deng R. Endoribonuclease activities of porcine reproductive and respiratory syndrome virus nsp11 was essential for nsp11 to inhibit IFN-beta induction. Mol Immunol. 2011;48(12-13):1568-72.

8. Posthuma CC, Nedialkova DD, Zevenhoven-Dobbe JC, Blokhuis JH, Gorbalenya AE, Snijder EJ. Site-directed mutagenesis of the Nidovirus replicative endoribonuclease NendoU exerts pleiotropic effects on the arterivirus life cycle. J Virol. 2006;80(4):1653-61.

9. Chen J, Shi X, Zhang X, Wang L, Luo J, Xing G, Deng R, Yang H, Li J, Wang A, et al. Porcine Reproductive and Respiratory Syndrome Virus (PRRSV) inhibits RNA-mediated gene silencing by targeting ago-2. Viruses. 2015; 7(10):5539-52

10. Wang C, Shi X, Zhang X, Wang A, Wang L, Chen J, Deng R, Zhang G. The endoribonuclease activity essential for the nonstructural protein 11 of porcine reproductive and respiratory syndrome virus to inhibit NLRP3 inflammasomemediated IL-1 beta induction. DNA Cell Biol. 2015;34(12):728-35.

11. Kim HS, Kwang J, Yoon IJ, Joo HS, Frey ML. Enhanced replication of porcine reproductive and respiratory syndrome (PRRS) virus in a homogeneous subpopulation of MA-104 cell line. Arch Virol. 1993;133(3-4):477-83.

12. Shi X, Zhang X, Wang F, Wang L, Qiao S, Guo J, Luo C, Wan B, Deng R, Zhang $\mathrm{G}$. The zinc-finger domain was essential for porcine reproductive and respiratory syndrome virus nonstructural protein-1alpha to inhibit the production of interferon-beta. J Interferon Cytokine Res. 2013;33(6):328-34.

13. Shi X, Qin L, LiU G, Zhao S, Peng N, Chen X. Dynamic balance of pSTAT1 and PSTAT3 in C57BL/6 mice infected with lethal or nonlethal Plasmodium yoelii. Cell Mol Immunol. 2008;5(5):341-8.

14. Beura LK, Sarkar SN, Kwon B, Subramaniam S, Jones C, Pattnaik AK, Osorio FA. Porcine reproductive and respiratory syndrome virus nonstructural protein 1 beta modulates host innate immune response by antagonizing IRF3 activation. J Virol. 2010;84(3):1574-84.

15. Richt JA, Lekcharoensuk P, Lager KM, Vincent AL, Loiacono CM, Janke BH, Wu WH, Yoon KJ, Webby RJ, Solorzano A, et al. Vaccination of pigs against swine influenza viruses by using an NS1-truncated modified live-virus vaccine. J Virol. 2006;80(22):11009-18.

16. Wacheck V, Egorov A, Groiss F, Pfeiffer A, Fuereder T, Hoeflmayer D, Kundi M, Popow-Kraupp T, Redlberger-Fritz M, Mueller CA, et al. A novel type of influenza vaccine: safety and immunogenicity of replication-deficient influenza virus created by deletion of the interferon antagonist NS1. J Infect Dis. 2010;201(3):354-62.

17. Zust R, Cervantes-Barragan L, Kuri T, Blakqori G, Weber F, Ludewig B, Thiel V. Coronavirus non-structural protein 1 is a major pathogenicity factor: implications for the rational design of coronavirus vaccines. PLoS Pathog. 2007;3(8):e109. 
18. Mahmood ur R, Ali I, Husnain T, Riazuddin S. RNA interference: the story of gene silencing in plants and humans. Biotechnol Adv. 2008;26(3):202-9.

19. Parashar D, Paingankar MS, Kumar S, Gokhale MD, Sudeep AB, Shinde SB, Arankalle VA. Administration of E2 and NS1 siRNAs inhibit chikungunya virus replication in vitro and protects mice infected with the virus. PLoS Negl Trop Dis. 2013;7(9), e2405.

20. Idrees $S$, Ashfaq $\cup A$, Khaliq $S$. RNAi: antiviral therapy against dengue virus. Asian Pac J Trop Biomed. 2013;3(3):232-6

21. Huang W, Li X, Yi M, Zhu S, Chen W. Targeted delivery of siRNA against hepatitis B virus by preS1 peptide molecular ligand. Hepatol Res. 2011;44(8): 897-906.

22. Kahana R, Kuznetzova L, Rogel A, Shemesh M, Hai D, Yadin H, Stram Y. Inhibition of foot-and-mouth disease virus replication by small interfering RNA. J Gen Virol. 2004;85(Pt 11):3213-7.

23. Wang Y, Liang Y, Han J, Burkhart KM, Vaughn EM, Roof MB, Faaberg KS Attenuation of porcine reproductive and respiratory syndrome virus strain MN184 using chimeric construction with vaccine sequence. Virology. 2008;371(2):418-29.

Submit your next manuscript to BioMed Central and we will help you at every step:

- We accept pre-submission inquiries

- Our selector tool helps you to find the most relevant journal

- We provide round the clock customer support

- Convenient online submission

- Thorough peer review

- Inclusion in PubMed and all major indexing services

- Maximum visibility for your research

Submit your manuscript at www.biomedcentral.com/submit
Biomed Central 